\title{
Mouthwash Use and Associated Factors among Saudi Adults: A Cross-sectional Study
}

\author{
Reem Al-kattan ${ }^{1 *}$, Eman Allam ${ }^{2,3}$, Majd Qadoumi ${ }^{4}$ and Nouf Alshibani ${ }^{1}$ \\ ${ }^{1}$ Department of Periodontics and Community Dentistry, College of Dentistry, King Saud University, Riyadh, Saudi Arabia \\ ${ }^{2}$ Department of Preventive Dental Sciences, College of Dentistry, Gulf Medical University, Ajman, UAE \\ ${ }^{3}$ Oral and Dental Research Division, National Research Centre, Cairo, Egypt \\ ${ }^{4}$ Dental Student, College of Dentistry, King Saud University, Riyadh, Saudi Arabia
}

*Corresponding author: Reem Al-Kattan BDS, MClinDent, Department of Periodontics and Community Dentistry, College of Dentistry, King Saud University, Riyadh, Saudi Arabia; Tel: +966505459855

Received: June 28, 2020; Accepted: July 06, 2020; Published: July 09, 2020

\begin{abstract}
The purpose of this study was to determine the state of mouthwash use, practice, and attitude among a cohort of adult Saudi population. A convenience sample of 999 outpatients was asked to participate in a self-administered survey on the mouthwash use, effects, attitude, and practice. While $38 \%$ of the participants reported that they never used a mouthwash, $14.4 \%, 28.7 \%$, and $18.8 \%$ used a mouthwash either daily, once every few days, or less than once a month, respectively. More than half of the respondents (55.4\%) trusted that the use of mouthwash does not cause any side effects and $70.5 \%$ indicated that they do not know whether using mouthwash would be considered a risk factor for oral cancer. Similarly, more than half of the respondents (50.4\%) were not aware of the active ingredients in a mouthwash. $69 \%$ indicated that the use of mouthwash does not compromise the importance of tooth brushing in plaque removal. There was significant difference in the practice and frequency of mouthwash use with regards to the social status, educational level, tooth brushing and flossing frequency, presence of caries, periodontal disease, and fixed restoration among respondents ( $\mathrm{p} \leq 0.05$ ). There were diverse patterns of knowledge and understanding regarding the proper and safe use of mouthwash among the studied sample.
\end{abstract}

Keywords: Mouthwash, Oral hygiene, Side effects, Oral health

\section{Introduction}

Mouthwashes are medicated solutions used as a supplement oral hygiene measure. Several oral conditions may require the use of a mouthwash ranging from halitosis, gingivitis and other periodontal diseases to treatment of ulcerative and infectious lesions and oral mucositis. Its ease of use in addition to the antibacterial effectiveness made mouthwashes a valuable preventive and therapeutic practice especially for periodontal diseases. A mouthwash may be recommended as an antimicrobial, anti-inflammatory, analgesic, deodorant, or astringent agent [1-3].

Oral Diseases, specifically caries and periodontitis, are a major public health concern owing to their high prevalence, incidence, and their effects on the individual's quality of life. The severe impact in terms of pain and suffering, impairment of function together with the high cost of treatment makes them also one of the leading health problems in most parts of the world, including Kingdom of Saudi Arabia (KSA). In 2012 the World Health Organization (WHO) estimated that, worldwide, $60-90 \%$ of school children and almost $100 \%$ adults would have dental problems [4-6].

The oral cavity harbors hundreds of microbial species that occur in both planktonic and biofilm forms. Poor oral hygiene leads to accumulation of oral bacteria and thus reducing the microbial load is considered the first step toward achieving good oral hygiene. This is usually achieved primarily through the use mechanical aids such as brushing and flossing. Studies have confirmed that the use of adjunct chemical measures such as mouthwashes has positive synergetic effect in improving the oral hygiene $[7,8]$. The aim of the current crosssectional study was to investigate the state of mouthwash use, practice, and attitude among a cohort of adult Saudi population.

\section{Materials and Methods}

The present cross-sectional study was carried out in the outpatient department of King Khaled University Hospital and the Dental Hospital, King Saud University in Riyadh, KSA. A convenience sample of 999 outpatients were asked to participate in the study. A self-administered structured questionnaire was distributed to the patients to evaluate the mouthwash use, effects, attitude, and practice. The inclusion criteria were adult patients above 18 years of age with no gender predilection and able to provide responses in the questionnaire form. The questionnaire was designed by the authors and was hand delivered to the respondents. Participation was voluntary and anonymous. The questionnaire included questions about the personal and demographic data as well as questions about the use of mouthwash, general oral health and oral hygiene practice. The study was reviewed and approved by the Research Ethics Review Committee (Research project no E-17-2744). The questionnaire was pre-tested on a representative sample of 35 subjects to check for appropriateness and any required modifications. The first 
seven questions determined subjects' demographic profile and included information on the gender, nationality, age, marital status, education, monthly income, and smoking status. The rest of the questions focused on type of dental care, oral hygiene practice, oral health conditions, and the use of mouthwashes.

All data were statistically analyzed using SPSS (Statistical Package for Social Science, IBM SPSS 21.0; Chicago, IL, USA). The data were subjected to a descriptive analysis and statistically represented in terms of numbers, percentages, and $95 \%$ confidence interval. Association between the parameters was done using crosstab chisquare test. Differences were considered statistically significant when the $\mathrm{p}$-values were $\leq 0.05$.

\section{Results}

The total number of adult subjects participating in the survey was 999 (394 males, 605 females, age range 18-65 years). Almost 75\% of the respondents were college educated or higher with half of them

Table 1: Demographic characteristics of the study sample ( $\mathrm{n}=999)$.

\begin{tabular}{|c|c|c|c|}
\hline Characteristics & & $\mathbf{n}$ & $\%$ \\
\hline \multicolumn{4}{|l|}{ Gender } \\
\hline & Male & 394 & $(39.40)$ \\
\hline & Female & 605 & $(60.60)$ \\
\hline \multicolumn{4}{|l|}{ Nationality } \\
\hline & Saudi & 943 & $(94.40)$ \\
\hline & Non-Saudi & 56 & $(5.60)$ \\
\hline \multicolumn{4}{|l|}{ Age } \\
\hline & $18-25 \mathrm{yrs}$ & 324 & $(32.40)$ \\
\hline & $26-35 \mathrm{yrs}$ & 386 & $(38.60)$ \\
\hline & $36-45$ yrs & 184 & $(18.40)$ \\
\hline & $46-55 \mathrm{yrs}$ & 92 & $(9.20)$ \\
\hline & $56-65 \mathrm{yrs}$ & 13 & $(1.30)$ \\
\hline \multicolumn{4}{|l|}{ Social Status } \\
\hline & Single & 404 & $(40.40)$ \\
\hline & Married & 561 & $(56.20)$ \\
\hline & Divorced & 24 & $(2.40)$ \\
\hline & Widow & 10 & $(1.00)$ \\
\hline \multicolumn{4}{|c|}{ Educational Level } \\
\hline & Uneducated & 2 & $(0.20)$ \\
\hline & Less than high school & 49 & $(4.90)$ \\
\hline & High school & 201 & $(20.10)$ \\
\hline & College & 619 & $(62.00)$ \\
\hline & Post graduate studies & 128 & $(12.80)$ \\
\hline \multicolumn{4}{|l|}{ Public \Private } \\
\hline & Public & 499 & $(49.90)$ \\
\hline & Private & 500 & $(50.10)$ \\
\hline \multicolumn{4}{|c|}{ Monthly Family Income } \\
\hline & Less than 5000 SAR & 86 & $(8.60)$ \\
\hline & 5000-10000 & 269 & $(26.90)$ \\
\hline & $10000-20000$ & 344 & (34.40) \\
\hline & More than 20000 & 300 & $(30.00)$ \\
\hline \multicolumn{4}{|l|}{ Smoking Status } \\
\hline & Currently Smoking & 158 & $(15.80)$ \\
\hline & $\begin{array}{l}\text { Previous Smoker (stopped within the last } 12 \\
\text { months) }\end{array}$ & 26 & $(2.60)$ \\
\hline & Previous Smoker (stopped over 12 months ago) & 34 & $(3.40)$ \\
\hline & Never Smoked & 781 & $(78.20)$ \\
\hline
\end{tabular}

(50.1\%) reporting a private educational institute. The sample almost composed of Saudi citizens (94.4\%). Demographic characteristics of the participants are shown in Table 1 .

Table 2 summarizes the oral hygiene practice and attitude among the study sample. Out of the 999 respondents, $68 \%$ had visited their dentist for regular dental care in less than a year. The reason for the visit was an emergency treatment in $38.5 \%$ of the cases while $25 \%$ and $15.8 \%$ were either for non-urgent treatment or dental checkup. Approximately $56 \%$ reported brushing their teeth twice or more a day while 33\%, 11\% either brushed once a day or less than daily, respectively. Similarly, $16.4 \%$ reported flossing daily while $27 \%, 16 \%, 40.5 \%$ flossed either once every few days, less than once a month, or never flossed, respectively.

Table 3 presents the general and oral health of the respondents. Almost half of the sample indicated good general and oral health, more than $70 \%$ being dentulous with more than 20 teeth. Caries was reported in $36 \%$, periodontal diseases in $20.7 \%$, oral ulcers in $4.8 \%$, staining in $8.7 \%$, halitosis in $21 \%$, and infections or abscesses in $5.6 \%$ of the sample. About half of the respondents (49\%) reported having a restoration or prosthetic appliances either removable, fixed, or implants.

Table 4 presents the responses regarding mouthwash use, knowledge, and practice. While $38 \%$ of the participants reported that they never used a mouthwash, $14.4 \%, 28.7 \%$, and $18.8 \%$ used a mouthwash either daily, once every few days, or less than once a month respectively. More than half of the respondents (55.4\%) trusted that the use of mouthwash does not cause any side effects and $70.5 \%$ indicated that they do not know whether using mouthwash would be considered a risk factor for oral cancer. Similarly, more than half

Table 2: Oral health practice and attitude among the study sample.

\begin{tabular}{|c|c|c|c|}
\hline Type of regular dental care & & $\mathbf{n}$ & $\%$ \\
\hline & Governmental Practice & 160 & $(16.00)$ \\
\hline & Private Practice & 585 & $(58.60)$ \\
\hline & None & 254 & $(25.40)$ \\
\hline \multicolumn{4}{|l|}{ Time since last dental check-up } \\
\hline & Less than a year & 679 & $(68.00)$ \\
\hline & $1-2$ years & 198 & $(19.80)$ \\
\hline & 2-5 years & 70 & $(7.00)$ \\
\hline & $5-10$ years & 29 & $(2.90)$ \\
\hline & Have not visited a dentist for over 10 years & 23 & $(2.30)$ \\
\hline \multicolumn{4}{|l|}{ Reasons for last dental visit } \\
\hline & $\begin{array}{l}\text { Emergency treatment needed for teeth or } \\
\text { gums }\end{array}$ & 385 & $(38.50)$ \\
\hline & Non-urgent treatment for teeth or gums & 251 & $(25.10)$ \\
\hline & Dental Check-up & 158 & $(15.80)$ \\
\hline & Can't remember & 61 & $(6.10)$ \\
\hline & Other & 144 & $(14.40)$ \\
\hline \multicolumn{4}{|l|}{ Tooth brushing frequency } \\
\hline & Twice or more a day & 558 & $(55.90)$ \\
\hline & Once a day & 329 & $(32.90)$ \\
\hline & Less than daily & 108 & $(10.80)$ \\
\hline & Never & 4 & $(0.40)$ \\
\hline \multicolumn{4}{|l|}{ Flossing Frequency } \\
\hline & Daily & 164 & $(16.40)$ \\
\hline & Once every few days & 271 & $(27.10)$ \\
\hline & Less than once a month & 159 & $(15.90)$ \\
\hline & Never & 405 & $(40.50)$ \\
\hline
\end{tabular}


Table 3: General and oral health characteristics of the study sample.

\begin{tabular}{|c|c|c|c|}
\hline General Health & & n & $\%$ \\
\hline & Excellent & 421 & $(42.10)$ \\
\hline & Good & 481 & $(48.10)$ \\
\hline & Fair & 82 & $(8.20)$ \\
\hline & Poor & 15 & $(1.50)$ \\
\hline \multicolumn{4}{|l|}{ Dental Health } \\
\hline & Excellent & 224 & $(22.40)$ \\
\hline & Good & 527 & $(52.80)$ \\
\hline & Fair & 196 & $(19.60)$ \\
\hline & Poor & 52 & $(5.20)$ \\
\hline \multicolumn{4}{|c|}{ Current number of natural teeth } \\
\hline & Fewer than 10 & 44 & $(4.40)$ \\
\hline & Between 10 and 19 & 255 & $(25.50)$ \\
\hline & 20 or more & 700 & $(70.10)$ \\
\hline \multicolumn{4}{|c|}{ Do you currently suffer of any of these conditions? } \\
\hline \multicolumn{4}{|l|}{ Caries } \\
\hline & Yes & 361 & $(36.10)$ \\
\hline & No & 525 & $(52.60)$ \\
\hline & Do not know & 113 & $(11.30)$ \\
\hline \multicolumn{4}{|c|}{ Periodontal Disease } \\
\hline & Yes & 207 & $(20.70)$ \\
\hline & No & 681 & $(68.20)$ \\
\hline & Do not know & 111 & $(11.10)$ \\
\hline \multicolumn{4}{|l|}{ Oral Ulcer } \\
\hline & Yes & 48 & $(4.80)$ \\
\hline & No & 846 & $(84.70)$ \\
\hline & Do not know & 105 & $(10.50)$ \\
\hline \multicolumn{4}{|l|}{ Stains } \\
\hline & Yes & 87 & $(8.70)$ \\
\hline & No & 815 & $(81.60)$ \\
\hline & Do not know & 97 & $(9.70)$ \\
\hline \multicolumn{4}{|l|}{ Halitosis } \\
\hline & Yes & 210 & $(21.00)$ \\
\hline & No & 677 & $(67.80)$ \\
\hline & Do not know & 112 & $(11.20)$ \\
\hline \multicolumn{4}{|c|}{ Infections (Abscess) } \\
\hline & Yes & 56 & $(5.60)$ \\
\hline & No & 857 & $(85.80)$ \\
\hline & Do not know & 86 & $(8.60)$ \\
\hline \multicolumn{4}{|c|}{ Do you currently have any of the following? } \\
\hline \multicolumn{4}{|l|}{ Bridges } \\
\hline & Yes & 269 & $(26.90)$ \\
\hline & No & 670 & $(67.10)$ \\
\hline & Do not know & 60 & $(6.00)$ \\
\hline \multicolumn{4}{|l|}{ Implants } \\
\hline & Yes & 149 & $(14.90)$ \\
\hline & No & 783 & $(78.40)$ \\
\hline & Do not know & 67 & $(6.70)$ \\
\hline \multicolumn{4}{|l|}{ Dentures } \\
\hline & Yes & 77 & $(7.70)$ \\
\hline & No & 848 & $(84.90)$ \\
\hline & Do not know & 74 & $(7.40)$ \\
\hline
\end{tabular}

of the respondents (50.4\%) were not aware of the active ingredients in a mouthwash. $69 \%$ indicated that the use of mouthwash does not compromise the importance of tooth brushing in plaque removal.

There was significant difference in the practice and frequency of
Table 4: Mouthwash use and practice among the study sample.

\begin{tabular}{|c|c|c|c|}
\hline How often do you rinse with a mouthwash & & n & $\%$ \\
\hline & Daily & 144 & $(14.40)$ \\
\hline & Once every few days & 287 & $(28.70)$ \\
\hline & Less than once a month & 188 & $(18.80)$ \\
\hline & Never & 380 & $(38.00)$ \\
\hline \multicolumn{4}{|l|}{ Does the use of mouthwashes have any side effects } \\
\hline & Yes & 106 & $(10.60)$ \\
\hline & No & 553 & $(55.40)$ \\
\hline & Do not know & 340 & $(34.00)$ \\
\hline \multicolumn{4}{|l|}{ Is the use of mouthwashes a risk factor for oral cancer } \\
\hline & Yes & 24 & $(2.40)$ \\
\hline & No & 271 & $(27.10)$ \\
\hline & Do not know & 704 & $(70.50)$ \\
\hline \multicolumn{4}{|l|}{$\begin{array}{l}\text { Are you aware of the different active ingredients } \\
\text { found in the mouthwashes }\end{array}$} \\
\hline & Yes & 121 & $(12.10)$ \\
\hline & No & 375 & $(37.50)$ \\
\hline & Do not know & 503 & $(50.40)$ \\
\hline \multicolumn{4}{|l|}{$\begin{array}{l}\text { Does the use of mouthwashes reduce the importance } \\
\text { of tooth brushing in plaque removal }\end{array}$} \\
\hline & Yes & 67 & $(6.70)$ \\
\hline & No & 690 & $(69.10)$ \\
\hline & Do not know & 242 & $(24.20)$ \\
\hline
\end{tabular}

mouthwash use with regards to the social status, educational level, the reason for last dental visit, tooth brushing and flossing frequency, caries, periodontal disease, and the presence of fixed restoration among respondents (Tables 5 and 6).

\section{Discussion}

Changing food habits along with the fast-paced modern lifestyle resulted in sharp rise of cavities and dental problems extensively. To combat these problems, adoption of oral hygiene practices that are easy to use and effective such as the use of mouthwashes seem to be a practical solution especially when mechanical aids are not sufficient to maintain optimum oral health. Studies have suggested that in combination with brushing, using an antimicrobial mouthwash could be more effective than flossing when it comes to preventing gingivitis. Some clinicians suggest that this is particularly important since at any given time, more than $50 \%$ of the public has gingivitis, and many with gingivitis may not even know they have it. Antimicrobial mouthwash help eliminate plaque-causing bacteria that brushing and flossing miss [9-12].

Generally, mouthwashes are classified into preventive, cosmetic, and therapeutic. Chemotherapeutic mouthwashes usually contain active ingredients that reduce inflammation. They also function as remineralizing agents, antimicrobial, astringent, analgesic, buffering, deodorizing to neutralize odor, or oxygenating cleansing action. Cosmetic mouthwashes can be used as fresheners or to reduce staining when it is superficial in unattached biofilm $[13,14]$.

The results of the current study indicated that, among the studied sample, mouthwash use was significantly associated with the frequency 
Table 5: Association of mouthwash use and practice with the demographics, socioeconomic and educational status, and smoking among the studied sample

\begin{tabular}{|c|c|c|c|c|c|c|c|c|c|c|c|}
\hline & \multirow{3}{*}{ Characteristics } & \multirow{3}{*}{ Total } & \multicolumn{8}{|c|}{ Responses } & \multirow{3}{*}{ P-value } \\
\hline & & & \multicolumn{2}{|c|}{ Daily } & \multicolumn{2}{|c|}{ Once every few days } & \multicolumn{2}{|c|}{ Less than once a month } & \multicolumn{2}{|c|}{ Never } & \\
\hline & & & Count (\%) & $95 \% \mathrm{CI}$ & Count (\%) & $95 \% \mathrm{CI}$ & Count (\%) & $95 \% \mathrm{CI}$ & Count (\%) & $95 \% \mathrm{CI}$ & \\
\hline \multicolumn{11}{|c|}{ Sex } & 0.083 \\
\hline & Male & 394 & $49(12.40)$ & $(9.54-16.07)$ & $102(25.90)$ & $(21.81-30.43)$ & $84(21.30)$ & $(17.56-25.63)$ & $159(40.40)$ & $(35.63-45.27)$ & \\
\hline & Female & 605 & $95(15.70)$ & $(13.02-18.81)$ & $185(30.60)$ & $(27.04-34.36)$ & $104(17.20)$ & $(14.39-20.40)$ & $221(36.50)$ & $(32.79-40.44)$ & \\
\hline \multirow[t]{7}{*}{ Age } & & & & & & & & & & & 0.415 \\
\hline & $18-25$ yrs & 324 & $48(14.80)$ & $(11.36-19.09)$ & $98(30.20)$ & $(25.50-35.46)$ & $55(17.00)$ & $(13.28-21.45)$ & $123(38.00)$ & $(32.85-43.36)$ & \\
\hline & $26-35$ yrs & 386 & $57(14.80)$ & $(11.58-18.66)$ & $116(30.10)$ & $(25.69-34.80)$ & $79(20.50)$ & $(16.75-24.78)$ & $134(34.70)$ & $(30.14-39.60)$ & \\
\hline & $36-45$ yrs & 184 & $28(15.20)$ & $(10.75-21.12)$ & $45(24.50)$ & $(18.81-31.15)$ & $32(17.40)$ & $(12.60-23.52)$ & $79(42.90)$ & $(35.99-50.15)$ & \\
\hline & $46-55$ yrs & 92 & $11(12.00)$ & $(6.81-20.16)$ & $26(28.30)$ & $(20.07-38.19)$ & $17(18.50)$ & $(11.87-27.62)$ & $38(41.30)$ & $(31.79-51.51)$ & \\
\hline & $56-65$ yrs & 11 & $0(0.00)$ & $(0.00-25.88)$ & $2(18.20)$ & $(5.14-47.70)$ & $5(45.50)$ & $(21.27-71.99)$ & $4(36.40)$ & $(15.16-64.62)$ & \\
\hline & $>65 \mathrm{yrs}$ & 2 & $0(0.00)$ & $(0.00-65.76)$ & $0(0.00)$ & $(0.00-65.76)$ & $0(0.00)$ & $(0.00-65.76)$ & $2(100.00)$ & $(34.24-100.0)$ & \\
\hline \multicolumn{11}{|c|}{ Social Status } & 0.012 \\
\hline & Single & 404 & $60(14.90)$ & $(11.71-18.65)$ & $125(30.90)$ & $(26.63-35.61)$ & $67(16.60)$ & $(13.27-20.52)$ & $152(37.60)$ & $(33.03-42.44)$ & \\
\hline & Married & 561 & $73(13.00)$ & $(10.48-16.05)$ & $150(26.70)$ & $(23.24-30.55)$ & $117(20.90)$ & $(17.70-24.41)$ & $221(39.40)$ & $(35.43-43.49)$ & \\
\hline & Divorced & 24 & $7(29.20)$ & $(14.92-49.17)$ & $11(45.80)$ & $(27.89-64.92)$ & $2(8.30)$ & $(2.31-25.84)$ & $4(16.70)$ & $(6.68-35.86)$ & \\
\hline & Widow & 10 & $4(40.00)$ & $(16.82-68.73)$ & $1(10.00)$ & $(1.79-40.41)$ & $2(20.00)$ & $(5.67-50.98)$ & $3(30.00)$ & $(10.78-60.32)$ & \\
\hline \multicolumn{11}{|c|}{ Educational Level } & 0.023 \\
\hline & Uneducated & 2 & $0(0.00)$ & $(0.00-65.76)$ & $1(50.00)$ & $(9.45-90.55)$ & $0(0.00)$ & $(0.00-65.76)$ & $1(50.00)$ & $(9.45-90.55)$ & \\
\hline & Less than high school & 49 & $9(18.40)$ & $(9.98-31.36)$ & $10(20.40)$ & $(11.48-33.64)$ & $7(14.30)$ & $(7.10-26.67)$ & $23(46.90)$ & $(33.70-60.62)$ & \\
\hline & High school & 201 & $35(17.40)$ & $(12.79-23.25)$ & $43(21.40)$ & $(16.29-27.57)$ & $32(15.90)$ & $(11.51-21.61)$ & $91(45.30)$ & $(38.54-52.18)$ & \\
\hline & College & 619 & $88(14.20)$ & $(11.69-17.19)$ & $190(30.70)$ & (27.19-34.43) & $115(18.60)$ & $(15.71-21.83)$ & $226(36.50)$ & $(32.81-40.38)$ & \\
\hline & Post graduate studies & 128 & $12(9.40)$ & (5.45-15.68) & $43(33.60)$ & $(25.99-42.14)$ & $34(26.60)$ & $(19.67-34.81)$ & $39(30.50)$ & $(23.16-38.92)$ & \\
\hline \multicolumn{11}{|c|}{ Monthly Family Income } & 0.879 \\
\hline & Less than $5000 \mathrm{SAR}$ & 86 & $16(18.60)$ & $(11.79-28.10)$ & $20(23.30)$ & $(15.59-33.21)$ & $16(18.60)$ & $(11.79-28.10)$ & $34(39.50)$ & $(29.86-50.10)$ & \\
\hline & 5000-10000 & 269 & $37(13.80)$ & $(10.14-18.38)$ & $76(28.30)$ & $(23.21-33.91)$ & $49(18.20)$ & $(14.07-23.27)$ & $107(39.80)$ & (34.11-45.73) & \\
\hline & $10000-20000$ & 344 & $43(12.50)$ & $(9.41-16.41)$ & $103(29.90)$ & $(25.34-34.98)$ & $66(19.20)$ & $(15.38-23.68)$ & $132(38.40)$ & $(33.39-43.61)$ & \\
\hline & More than 20000 & 300 & $48(16.00)$ & $(12.29-20.57)$ & $88(29.30)$ & $(24.47-34.72)$ & $57(19.00)$ & $(14.96-23.82)$ & $107(35.70)$ & $(30.46-41.24)$ & \\
\hline \multicolumn{11}{|c|}{ Smoking Status } & 0.770 \\
\hline & Currently Smoking & 158 & $26(16.50)$ & $(11.49-23.02)$ & $47(29.70)$ & $(23.17-37.29)$ & $24(15.20)$ & $(10.43-21.61)$ & $61(38.60)$ & $(31.37-46.39)$ & \\
\hline & Previous Smoker (stopped within the last 12 months) & 26 & $4(15.40)$ & $(6.15-33.53)$ & $5(19.20)$ & $(8.51-37.88)$ & $8(30.80)$ & $(16.50-49.99)$ & $9(34.60)$ & $(19.42-53.78)$ & \\
\hline & Previous Smoker (stopped over 12 months ago) & 34 & $3(8.80)$ & $(3.04-22.96)$ & $10(29.40)$ & $(16.83-46.17)$ & $8(23.50)$ & $(12.44-40.00)$ & $13(38.20)$ & $(23.90-54.96)$ & \\
\hline & Never Smoked & 781 & $111(14.20)$ & $(11.94-16.83)$ & $225(28.80)$ & $(25.74-32.08)$ & $148(19.00)$ & $(16.36-21.85)$ & $297(38.00)$ & $(34.69-41.49)$ & \\
\hline
\end{tabular}


Table 6: Association of mouthwash use and practice with the oral health and oral hygiene practices among the studied sample

\begin{tabular}{|c|c|c|c|c|c|c|c|c|c|c|}
\hline \multirow{3}{*}{ Characteristics } & \multirow{3}{*}{ Total } & \multicolumn{8}{|c|}{ Responses } & \multirow{3}{*}{ P-value } \\
\hline & & \multicolumn{2}{|c|}{ Daily } & \multicolumn{2}{|c|}{ Once every few days } & \multicolumn{2}{|c|}{ Less than once a month } & \multicolumn{2}{|c|}{ Never } & \\
\hline & & Count (\%) & $95 \% \mathrm{CI}$ & Count (\%) & $95 \% \mathrm{CI}$ & Count (\%) & $95 \% \mathrm{CI}$ & Count (\%) & $95 \% \mathrm{CI}$ & \\
\hline \multicolumn{10}{|c|}{ Reasons for last dental visit } & 0.025 \\
\hline Emergency treatment needed for teeth or gums & 385 & $49(12.70)$ & $(9.76-16.43)$ & $111(28.80)$ & $(24.53-33.55)$ & $76(19.70)$ & $(16.07-24.01)$ & $149(38.70)$ & $(33.97-43.65)$ & \\
\hline Non-urgent treatment for teeth or gums & 251 & $38(15.10)$ & $(11.23-20.10)$ & $81(32.30)$ & $(26.79-38.28)$ & $44(17.50)$ & $(13.33-22.71)$ & $88(35.10)$ & $(29.42-41.15)$ & \\
\hline Dental Check-up & 158 & $23(14.60)$ & $(9.90-20.90)$ & $46(29.10)$ & $(22.59-36.62)$ & $31(19.60)$ & $(14.18-26.50)$ & $58(36.70)$ & $(29.59-44.46)$ & \\
\hline Can't remember & 61 & $11(18.00)$ & $(10.38-29.47)$ & $9(14.80)$ & $(7.96-25.72)$ & $4(6.60)$ & $(2.58-15.69)$ & $37(60.70)$ & $(48.12-71.94)$ & \\
\hline Other & 144 & $23(16.00)$ & $(10.88-22.83)$ & $40(27.80)$ & $(21.11-35.60)$ & $33(22.90)$ & $(16.81-30.44)$ & $48(33.30)$ & $(26.15-41.37)$ & \\
\hline \multicolumn{10}{|c|}{ Flossing Frequency } & 0.001 \\
\hline Daily & 164 & $50(30.50)$ & $(23.96-37.92)$ & $52(31.70)$ & $(25.08-39.18)$ & $16(9.80)$ & $(6.10-15.26)$ & $46(28.00)$ & $(21.74-35.37)$ & \\
\hline Once every few days & 271 & $81(29.90)$ & $(24.75-35.59)$ & $100(36.90)$ & $(31.38-42.79)$ & $47(17.30)$ & $(13.30-22.30)$ & $43(15.90)$ & $(12.00-20.69)$ & \\
\hline Less than once a month & 159 & $59(37.10)$ & $(29.99-44.84)$ & $42(26.40)$ & $(20.18-33.77)$ & $42(26.40)$ & $(20.18-33.77)$ & $16(10.10)$ & $(6.29-15.72)$ & \\
\hline Never & 405 & $190(46.90)$ & $(42.10-51.78)$ & $93(23.00)$ & $(19.13-27.30)$ & $83(20.50)$ & $(16.84-24.69)$ & $39(9.60)$ & $(7.12-12.89)$ & \\
\hline \multicolumn{10}{|c|}{ Tooth brushing frequency } & 0.001 \\
\hline Twice or more a day & 558 & $167(29.90)$ & $(26.28-33.86)$ & $174(31.20)$ & $(27.48-35.14)$ & $103(18.50)$ & $(15.46-21.89)$ & $114(20.40)$ & $(17.29-23.97)$ & \\
\hline Once a day & 329 & $154(46.80)$ & $(41.49-52.21)$ & $93(28.30)$ & $(23.68-33.37)$ & $58(17.60)$ & $(13.89-22.11)$ & $24(7.30)$ & $(4.95-10.62)$ & \\
\hline Less than daily & 108 & $57(52.80)$ & $(43.43-61.94)$ & $18(16.70)$ & $(10.81-24.82)$ & $27(25.00)$ & $(17.79-33.93)$ & $6(5.60)$ & $(2.57-11.60)$ & \\
\hline Never & 4 & $2(50.00)$ & $(15.00-85.00)$ & $2(50.00)$ & $(15.00-85.00)$ & $0(0.00)$ & $(0.00-48.99)$ & $0(0.00)$ & $(0.00-48.99)$ & \\
\hline \multicolumn{10}{|c|}{ Caries } & 0.001 \\
\hline Yes & 361 & $147(40.70)$ & $(35.78-45.86)$ & $93(25.80)$ & $(21.52-30.51)$ & $68(18.80)$ & $(15.14-23.19)$ & $53(14.70)$ & $(11.40-18.70)$ & \\
\hline No & 525 & $176(33.50)$ & $(29.61-37.66)$ & $169(32.20)$ & $(28.34-36.30)$ & $95(18.10)$ & $(15.04-21.62)$ & $85(16.20)$ & $(13.29-19.58)$ & \\
\hline Do not know & 113 & $57(50.40)$ & $(41.36-59.49)$ & $25(22.10)$ & $(15.46-30.62)$ & $25(22.10)$ & $(15.46-30.62)$ & $6(5.30)$ & $(2.46-11.10)$ & \\
\hline \multicolumn{10}{|c|}{ Periodontal Disease } & 0.001 \\
\hline Yes & 207 & $58(28.00)$ & $(22.35-34.50)$ & $73(35.30)$ & $(29.08-41.99)$ & $37(17.90)$ & $(13.25-23.66)$ & $39(18.80)$ & $(14.10-24.72)$ & \\
\hline No & 681 & $272(39.90)$ & $(36.33-43.67)$ & $196(28.80)$ & $(25.51-32.29)$ & $117(17.20)$ & $(14.53-20.20)$ & $96(14.10)$ & $(11.69-16.92)$ & \\
\hline Do not know & 111 & $50(45.00)$ & $(36.11-54.32)$ & $18(16.20)$ & $(10.51-24.19)$ & $34(30.60)$ & $(22.82-39.73)$ & $9(8.10)$ & $(4.33-14.70)$ & \\
\hline \multicolumn{10}{|c|}{ Oral Ulcer } & 0.336 \\
\hline Yes & 48 & $8(16.70)$ & $(8.70-29.58)$ & $13(27.10)$ & $(16.56-40.99)$ & $5(10.40)$ & $(4.53-22.17)$ & $22(45.80)$ & $(32.57-59.71)$ & \\
\hline No & 846 & $121(14.30)$ & $(12.10-16.82)$ & $252(29.80)$ & $(26.81-32.96)$ & $159(18.80)$ & $(16.30-21.56)$ & $314(37.10)$ & $(33.93-40.43)$ & \\
\hline Do not know & 105 & $15(14.30)$ & $(8.86-22.24)$ & $22(21.00)$ & $(14.26-29.69)$ & $24(22.90)$ & $(15.87-31.76)$ & $44(41.90)$ & (32.91-51.46) & \\
\hline
\end{tabular}




\begin{tabular}{|c|c|c|c|c|c|c|c|c|c|c|}
\hline \multicolumn{10}{|c|}{ Stains } & 0.709 \\
\hline Yes & 87 & $13(14.90)$ & $(8.94-23.90)$ & $26(29.90)$ & $(21.29-40.19)$ & $14(16.10)$ & $(9.83-25.21)$ & $34(39.10)$ & $(29.50-49.59)$ & \\
\hline No & 815 & $118(14.50)$ & $(12.23-17.06)$ & $239(29.30)$ & $(26.31-32.55)$ & $150(18.40)$ & $(15.89-21.21)$ & $308(37.80)$ & $(34.53-41.17)$ & \\
\hline Do not know & 97 & $13(13.40)$ & $(8.00-21.59)$ & $22(22.70)$ & $(15.48-31.96)$ & $24(24.70)$ & $(17.23-34.18)$ & $38(39.20)$ & $(30.06-49.13)$ & \\
\hline \multicolumn{10}{|c|}{ Smell } & 0.188 \\
\hline Yes & 210 & $34(16.20)$ & $(11.82-21.77)$ & $54(25.70)$ & $(20.27-32.02)$ & $44(21.00)$ & $(15.99-26.95)$ & $78(37.10)$ & $(30.89-43.85)$ & \\
\hline No & 677 & $100(14.80)$ & $(12.30-17.64)$ & $205(30.30)$ & $(26.94-33.84)$ & $116(17.10)$ & $(14.48-20.15)$ & $256(37.80)$ & (34.24-41.52) & \\
\hline Do not know & 112 & $10(8.90)$ & $(4.92-15.66)$ & $28(25.00)$ & $(17.90-33.76)$ & $28(25.00)$ & $(17.90-33.76)$ & $46(41.10)$ & $(32.40-50.33)$ & \\
\hline \multicolumn{10}{|c|}{ Infections (Abscess) } & 0.448 \\
\hline Yes & 56 & $8(14.30)$ & $(7.42-25.74)$ & $20(35.70)$ & $(24.45-48.80)$ & $8(14.30)$ & $(7.42-25.74)$ & $20(35.70)$ & $(24.45-48.80)$ & \\
\hline No & 857 & $126(14.70)$ & $(12.49-17.23)$ & $249(29.10)$ & $(26.11-32.18)$ & $161(18.80)$ & $(16.32-21.54)$ & $321(37.50)$ & $(34.28-40.75)$ & \\
\hline Do not know & 86 & $10(11.60)$ & $(6.44-20.10)$ & $18(20.90)$ & $(13.67-30.68)$ & $19(22.10)$ & $(14.62-31.94)$ & $39(45.30)$ & $(35.25-55.84)$ & \\
\hline \multicolumn{10}{|c|}{ Others } & 0.495 \\
\hline Yes & 112 & $17(15.20)$ & $(9.70-22.97)$ & $33(29.50)$ & $(21.81-38.47)$ & $20(17.90)$ & $(11.87-25.98)$ & $42(37.50)$ & $(29.09-46.74)$ & \\
\hline No & 733 & $109(14.90)$ & $(12.48-17.63)$ & $216(29.50)$ & $(26.28-32.87)$ & $141(19.20)$ & $(16.55-22.25)$ & $267(36.40)$ & $(33.03-39.98)$ & \\
\hline Do not know & 154 & $18(11.70)$ & $(7.52-17.72)$ & $38(24.70)$ & $(18.54-32.05)$ & $27(17.50)$ & $(12.34-24.30)$ & $71(46.10)$ & $(38.42-53.97)$ & \\
\hline \multicolumn{10}{|c|}{ Bridges } & 0.037 \\
\hline Yes & 269 & $96(35.70)$ & $(30.20-41.58)$ & $73(27.10)$ & $(22.18-32.75)$ & $55(20.40)$ & $(16.06-25.67)$ & $45(16.70)$ & $(12.74-21.65)$ & \\
\hline No & 670 & $257(38.40)$ & $(34.75-42.10)$ & $207(30.90)$ & $(27.52-34.50)$ & $117(17.50)$ & $(14.77-20.52)$ & $89(13.30)$ & $(10.92-16.06)$ & \\
\hline Do not know & 60 & $27(45.00)$ & $(33.09-57.51)$ & $7(11.70)$ & $(5.77-22.18)$ & $16(26.70)$ & $(17.14-39.01)$ & $10(16.70)$ & $(9.32-28.04)$ & \\
\hline \multicolumn{10}{|c|}{ Implants } & 0.171 \\
\hline Yes & 149 & $26(17.40)$ & $(12.20-24.34)$ & $51(34.20)$ & $(27.09-42.16)$ & $22(14.80)$ & $(9.96-21.35)$ & $50(33.60)$ & $(26.48-41.47)$ & \\
\hline No & 783 & $105(13.40)$ & $(11.20-15.98)$ & $223(28.50)$ & $(25.43-31.74)$ & $151(19.30)$ & $(16.67-22.19)$ & $304(38.80)$ & $(35.48-42.29)$ & \\
\hline Do not know & 67 & $13(19.40)$ & $(11.70-30.42)$ & $13(19.40)$ & $(11.70-30.42)$ & $15(22.40)$ & $(14.07-33.71)$ & $26(38.80)$ & $(28.05-50.78)$ & \\
\hline \multicolumn{10}{|c|}{ Removable } & 0.762 \\
\hline Yes & 77 & $15(19.50)$ & $(12.18-29.68)$ & $18(23.40)$ & $(15.33-33.96)$ & $15(19.50)$ & $(12.18-29.68)$ & $29(37.70)$ & (27.67-48.82) & \\
\hline No & 848 & $117(13.80)$ & $(11.64-16.29)$ & $250(29.50)$ & $(26.51-32.64)$ & $157(18.50)$ & $(16.04-21.26)$ & $324(38.20)$ & $(35.00-41.53)$ & \\
\hline Do not know & 74 & $12(16.20)$ & $(9.53-26.24)$ & $19(25.70)$ & $(17.10-36.66)$ & $16(21.60)$ & $(13.77-32.27)$ & $27(36.50)$ & $(26.44-47.87)$ & \\
\hline
\end{tabular}


of tooth brushing and flossing. Patients who regularly brush their teeth and use a dental floss were keener to ensure sufficient oral hygiene by also using a mouthwash. Lang et al., [15] reported that the use of antimicrobial mouthwash for 30 seconds once a day as an adjunct to daily tooth brushing reduced gingivitis and caries incidence within six months. Other reports suggested that although using mouthwashes as antimicrobial agents have a good potential in controlling gingivitis, their regular use can cause significant adverse effects like teeth staining and drug resistance [16]. This highlight the importance of evaluating the risk/benefits of recommending a mouthwash by the dental practitioner and that the advice should be tailored and modified case by case.

In regard to the association of mouthwash use and the demographic factors, only social and educational status showed significant association with the frequency of the mouthwash use among the study sample. Contrary to the expectation, mouthwash use was not significantly different in relation to other important demographic criteria such as gender, age or even the smoking status. It is always predictable that oral hygiene practices are more significant in females who are potentially more attentive to appearance. It was also assumed that smokers would necessarily report more mouthwash use than nonsmokers. However, this could be related to the small sample size in the current study that is also should only hardly be considered representative to the whole community as the respondents were all recruited from only two outpatients' clinics.

The results of the present study also indicated that more than $89 \%$ of the respondents who used mouthwash either did not know or declined the fact that mouthwash use may have any side effects. Similarly, 97\% indicated that they do not believe mouthwash use can present a risk factor for development of oral cancer. In addition, almost $88 \%$ of the respondents did not know the active ingredient of a mouthwash. Whether the patient is aware of or not, clinicians need to make careful recommendation on mouthwashes based on several factors that include most importantly the efficacy and safety of the mouthwash. The selection of the right mouthwash recommendation majorly depends on the patients' oral condition as well as the ability to perform good oral hygiene practice especially brushing and flossing. Active ingredient is another factor to consider since some precautions need to be considered. For example, xerostomia could be worsened by the drying effect of an alcohol containing mouthwash.

The results showed significant difference in the frequency of mouthwash use when patients reported existing caries and periodontal diseases which indicate that the presence of chronic oral health conditions would encourage patients to seek additional oral hygiene means such as using mouthwashes. These results were in line with those reported by other investigators $[3,17,18]$. The presence of fixed restoration such as dental bridges also seemed to be a trigger to use mouthwash, most probably because those patients are traditionally advised on the importance of maintaining excellent oral hygiene and then using mouthwash could be recommended to facilitate cleaning the areas that are inaccessible to tooth brushing and flossing.

In conclusion, mouthwashes are formulated for a variety of oral benefits including mouth freshening, prevention of caries, biofilm control, and control of odor. Several factors must be considered when making a mouthwash recommendation including whether the patient is currently able to control biofilm through other methods and whether they may consider rinsing a substitute for another mechanical procedures such as brushing and flossing. It is important that target populations receive oral health advice that is tailored to meet their individual needs. These messages may need to be adapted and the influence on these target groups has to be taken into account. It is thus very important to understand the behavior, knowledge, and attitude of any community group toward the oral hygiene practices including mouthwash use. A targeted and tailored health education advice on the proper oral hygiene practice is a significant, cost-effective strategy to reduce the burden of oral disease and maintain oral health and quality of life.

\section{Acknowledgement}

The authors would like to thank the College of Dentistry Research Centre and Deanship of Scientific Research at King Saud University, Saudi Arabia, for funding this research project.

\section{Reference}

1. Eley BM (1999) Periodontology: Antibacterial agents in the control of supragingival plaque. Br Dent J 186: 286-296. [crossref]

2. Moran JM (2008) Home-use oral hygiene products: Mouthrinses. Periodontol 2000. 48:42-53. [crossref]

3. Macfarlane TV, Kawecki MM, Cunningham C, Bovarid I, Morgan R et al. (2011) Mouthwash use in general population: Results from adult dental health survey in Grampian, Scotland. J Oral Maxillofac Res. 1: 2. [crossref]

4. Petersen PE (2003) The World Oral Health Report 2003: Continuous improvement of oral health in the 21st century - The approach of the WHO Global Oral Health Programme. Community Dent Oral Epidemiol 1:3-23. [crossref]

5. WHO (2012) Media Centre-Oral Health. World Health Organization. April, 2012.

6. Haerian-Ardakani A, Rezaei M, Talebi-Ardakani M, Valian NK, Amid R et al. (2015) Comparison of antimicrobial effects of three different mouthwashes. Iranian Journal of Public Health 44: 997-1003. [crossref]

7. CharlesCH, Mostler KM, Bartels LL, Mankodi SM (2004) Comparative antiplaque and antigingivitis effectiveness of a chlorhexidine and an essential oil mouthrinse: 6-month clinical trial. J Clin Periodontol 31: 878-884. [crossref]

8. Berger D, Rakhamimova A, Pollack A, Loewy Z (2018) Oral biofilms: Development, control, and analysis. High Throughput 7: 24. [crossref]

9. Loe H (2000) Oral hygiene in the prevention of caries and periodontal disease International Dental Journal 50: 129-139. [crossref]

10. Bauroth K, Charles CH, Mankodi SM, Simmons K, Zhao Q et al. (2003) The efficacy of an essential oil antiseptic mouthrinse vs. dental floss in controlling interproximal gingivitis: a comparative study. The Journal of the American Dental Association 134: 359-365. [crossref]

11. Barnett ML (2006) The rationale for the daily use of an antimicrobial mouthrinse. The Journal of the American Dental Association 137: 16-21. [crossref]

12. Van Der Weijden, F, Slot DE (2011) Oral hygiene in the prevention of periodontal diseases: the evidence. Periodontology 2000 55: 104-123. [crossref]

13. Tuzun B, Firatli S, Tüzün Y, Firatli E, Wolf R (2001) Oral therapeutics and oral cosmetics. Clinics Dermatol 19: 449-51. [crossref]

14. Vranic E, Lacevic A, Mehmedagic A, Uzunovic A (2004) Formulation ingredients for toothpastes and mouthwashes. BJBMS 4: 51. [crossref]

15. Lang NP, Hotz P, Graf H, Geering AH, Saxer UP et al. (1982) Effects of supervised chlorhexidine mothrinses in children. A longitudinal clinical trial. J Periodontal Res 17:101-11. [crossref] 
Reem Al-Kattan (2020) Mouthwash Use and Associated Factors among Saudi Adults: A Cross-sectional Study

16. Jothika M, Vanajassun PP, Someshwar B (2015) Effectiveness of probiotic, chlorhexidine and fluoride mouthwash against Streptococcus mutans Randomized, single-blind, in vivo study. J Int Soc Prev Community Dent 5: 44-48. [crossref]
17. Farah CS, Mclntosh L, McCullough MJ (2009) Mouthwashes. Aust Prescr 32: 162-164.

18. Huskinson W, Lloyd H (2009) Oral health in hospitalized patients: assessment and hygiene. Nursing Standard 23: 43-47. [crossref]

\section{Citation:}

Reem Al-Kattan, Eman Allam, Majd Qadoumi and Nouf Alshibani (2020) Mouthwash Use and Associated Factors among Saudi Adults: A Cross-sectional Study. J Dent Maxillofacial Res Volume 3(2): 1-8. 